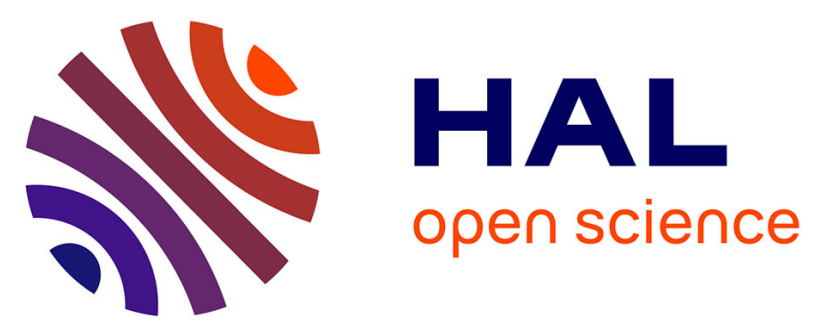

\title{
Neutron flux monitoring with in-vessel fission chambers to detect an inadvertent control rod withdrawal in a sodium-cooled fast reactor
}

\author{
V. Verma, C. Jammes, C. Hellesen, P. Filliatre, S. Jacobsson Svärd
}

\section{- To cite this version:}

V. Verma, C. Jammes, C. Hellesen, P. Filliatre, S. Jacobsson Svärd. Neutron flux monitoring with invessel fission chambers to detect an inadvertent control rod withdrawal in a sodium-cooled fast reactor. Annals of Nuclear Energy, 2016, 94, pp.487-493. 10.1016/j.anucene.2016.04.019 . cea-02380833

HAL Id: cea-02380833

https://hal-cea.archives-ouvertes.fr/cea-02380833

Submitted on 26 Nov 2019

HAL is a multi-disciplinary open access archive for the deposit and dissemination of scientific research documents, whether they are published or not. The documents may come from teaching and research institutions in France or abroad, or from public or private research centers.
L'archive ouverte pluridisciplinaire HAL, est destinée au dépôt et à la diffusion de documents scientifiques de niveau recherche, publiés ou non, émanant des établissements d'enseignement et de recherche français ou étrangers, des laboratoires publics ou privés. 


\title{
Neutron flux monitoring with in-vessel fission chambers to detect inadvertent control rod withdrawal in Sodium-cooled fast reactor
}

\author{
V. Verma ${ }^{\text {b,a }}$, C. Jammes ${ }^{\text {a,* }}$, C. Hellesen ${ }^{\mathrm{b}}$, P. Filliatre ${ }^{\mathrm{a}}$ \\ S. Jacobsson Svärd ${ }^{b}$ \\ ${ }^{a} C E A, D E N$, DER, Instrumentation Sensors and Dosimetry Laboratory, \\ Cadarache, F-13108 St-Paul-lez-Durance, France. \\ ${ }^{\mathrm{b}}$ Division of Applied Nuclear Physics, Uppsala University, Box 516, SE-75120 \\ Uppsala, Sweden
}

\begin{abstract}
Neutron flux monitoring system forms an integral part of the safety design of a Generation IV sodium cooled fast reactor system. During the initial design phase of a neutron flux monitoring system, one needs to explore various locations and configurations. Diverse possibilities of detector system installation should be studied for different locations in the reactor vessel in order to detect any perturbations in the core. In this paper, we investigate the possibility of placing fission chambers beyond the lateral neutron shield, ex-core but in-vessel and study the detectability of inadvertent control rod withdrawal with these fission chambers. A generic core design of a Generation IV, 1500 MWth French sodium-cooled fast reactor is used for the study and calculations are performed with the Monte Carlo code SERPENT2. We propose certain design changes that are needed to be incorporated, w.r.t. the facilitation of neutron transport to this ex-core location.

We are able to show that there is a visible signature in the fission chambers following an inadvertent control rod withdrawal in the core. The equally-spaced detectors azimuthally, are able to follow changes in the neutron flux distribution in the core. This study helps us analyze multiple detector locations and give general trends for monitoring indications to detect any perturbation in the core.
\end{abstract}

Key words: Protection systems, safety, accidents, Instrumentation, Sodium Cooled Fast reactors, Fission chambers

PACS: 28.41.Te, 28.41.Rc, 28.50.Ft, 29.40.Cs

* Corresponding author - Phone +33-4-4225-7151

Email address: christian.jammes@cea.fr (C. Jammes). 


\section{Introduction}

The French Atomic Energy and Alternative Energies Commission (CEA), EDF and AREVA French partners run a coordinated research program on Generation IV (Gen-IV) Sodium-cooled Fast Reactors (SFRs) within the framework of the French Act, dated June 28th 2006, since 2007. Safety and reliability are essential needs for development and operation of Gen-IV SFRs. Development of a neutron flux monitoring system (NFMS) for the French sodiumcooled fast reactor is one of the R\&D areas identified within this program. There is a need to confidently detect incidents in their earliest possible stages [1]. Traditionally, SFRs use a combination of high temperature fission chambers and proportional counters at various locations outside the core. For the purpose of the French Gen-IV program, fission chambers have been identified as the best choice for monitoring the core in all states of reactor operations $[2]$.

Several initiating events that may lead to incidental or accidental situations have been identified. Based on previous studies [1], an inadvertent control rod withdrawal (IRW) is considered to be a probable and penalizing event. An IRW would introduce a positive reactivity and change the power profile of the core. It may eventually lead to a local core melting accident. Although the likelihood of such an event is reduced by design as much as possible, the NFMS has to be able to confidently detect its possible occurrence.

In this paper we investigate the possibility of using a set of in-vessel, ex-core, fission chambers to detect the spatial changes in the power profile that would result from an IRW. A preliminary core design of a Generation IV, 1500 MWth sodium-cooled fast reactor (SFR) designed at CEA [3] is used for our study. We use the Monte Carlo based code SERPENT2 [4] in criticality mode for the neutron transport calculations. The paper is organized as follows: We review NFMS objectives and limitations in Section 2. This is followed by reactor core description, choice of detectors and their location in Section 3.1 to 3.3. We present the calculation route and the computational tools used in Section 3.4. We show the results from the criticality calculations in Section 4 followed by a discussion in Section 5 and conclusion in Section 6 .

\section{Neutron flux monitoring system}

The neutron flux monitoring system plays an important role in the prevention of incidents and accidents as well as in the regulation of the overall reactor power and reactivity [1]. The NFMS must detect at the earliest, any drifts and irregularities with regards to the normal operating range.

In a Sodium-cooled fast reactor (SFR), it is a challenge to place the detectors of the system in-core as there are high fluxes of neutrons (up to $10^{15} \mathrm{~cm}^{-2} \mathrm{~s}^{-1}$ ), 
and high temperature conditions. Previous studies made at CEA have shown that high temperature fission chambers (HTFCs) are promising detector candidates in the NFMS since they can provide reliable in-vessel measurements in SFRs for about 10 years of operation $[5,6]$. This wide range capability of HTFCs comes from the fact that a fission chamber can operate in pulse mode at low count rate and Campbelling mode at high count rate. In HTFCs, there is a sufficient overlap of the signals in pulse and campbelling modes in order to ensure the linearity of the neutron flux measurement [7].

The high neutron flux inside the core can deplete the fissile layer of fission chambers quickly. This harsh environment and compact design of SFR cores does not permit an in-core detector installation, however, in-vessel installation outside the core is feasible. The current possible ex-core regions for detector installation are: the lower part of the above-core structure (ACS), the region beyond the radial neutron shield and the core support structure (CSS) [1]. One of the main drawbacks associated with ACS is that the detector signal is cut off during fuel handling operations as the above core structure moves along with the rotatable plugs. This explains why SFRs need a combination of detectors at different locations for a consolidated monitoring system.

In this paper, the lateral installation of fission chambers is studied. The detectors installed in such radial locations might need a neutron channel in the radial neutron shield assemblies to allow for a sufficiently intense neutron flux to reach the fission chambers. Such arrangement has previously been used in Superphenix as well. The neutron channel design is discussed in more detail in Section 3.2. Throughout this paper, fission chambers and detectors are used synonymously.

\section{$3 \quad$ Neutron transport modelling}

\subsection{Core neutronics features}

A generic core design of a Generation IV, 1500 MWth sodium-cooled fast reactor (SFR) designed at CEA [3] is used for the present study. The design has an evolutionary axially heterogeneous, low void effect core (CFV) loaded with MOX fuel. The CFV core focuses on optimizing the reactivity coefficients so as to obtain improved core behavior during accidental conditions such as Loss of Flow. The fissile zone is divided in two parts radially, having different heights. By making the outer core higher than the inner one, the surface of neutron leakage is enhanced, thereby increasing the leakage in the upper sodium plenum. Axially, there is an internal fertile blanket which allows for an increased flux towards the upper fissile zone. Thus, the CFV core which has a negative void coefficient, maximizes neutron leakage from the core in 
the event of an accident and reduces the core reactivity in case of coolant temperature increase. This is an improvement on the previous Superphenix core where a sodium void resulted in higher core reactivity. Out of the 18 control rods, 12 are regulating rods (CSD) and 6 are shutdown rods (DSD) with $\mathrm{B}_{4} \mathrm{C}$ absorber pins. CSDs are inserted into the core during normal operation for flux flattening and reactivity control while DSDs are inserted only in the event of an accidental scram. Figure 1 shows the model of the SFR core used in SERPENT. We modelled the core accurately with the axial heterogeneous description. The reflector and the neutron shields have homogeneous compositions.

\subsection{Installation of fission chambers}

In the model presented in this paper, neutron channels are used to enhance the transport of neutrons from the core to the detectors. The channels are tapered to reduce unnecessary leakage and activation outside the core, and they are located symmetrically around the neutron shield at two axial locations. In this paper we chose two axial locations for the detectors as a first survey of suitable detector positions. Since the core under study is axially strongly in-homogenous due to the internal fertile blanket, it is not obvious where the optimal detector location for detection of a control rod withdrawal is. The two chosen locations coincide with the lower and upper fissile zones of the core.

The tapered design is formed by leaving out an intermediate region filled with sodium in three radially consecutive shield subassemblies. The sodium filled regions decrease in height as we move outwards. Finally, two sets of six equally-spaced U-235 fission chambers each are placed at the end of each neutron channel giving 6 angular detectors around the core at two axial locations. The detectors are located at a radial distance of about $285 \mathrm{~cm}$ from the core center. Throughout this paper, we refer to the two detector sets as the lower and upper detectors respectively. The arrangement is illustrated with a top view and an axial cross-section of the core in Figures 1 and 2, respectively. A detailed view of the neutron channel is also shown in Figure 3.

\subsection{Inadvertent control rod withdrawal}

The detector responses during the control rod withdrawal are calculated in a series of withdrawal steps ( $0 \% 25 \% 50 \% 75 \%$ and $100 \%)$ from the bottom of the core up to the control rod's parking position, which is just above the 


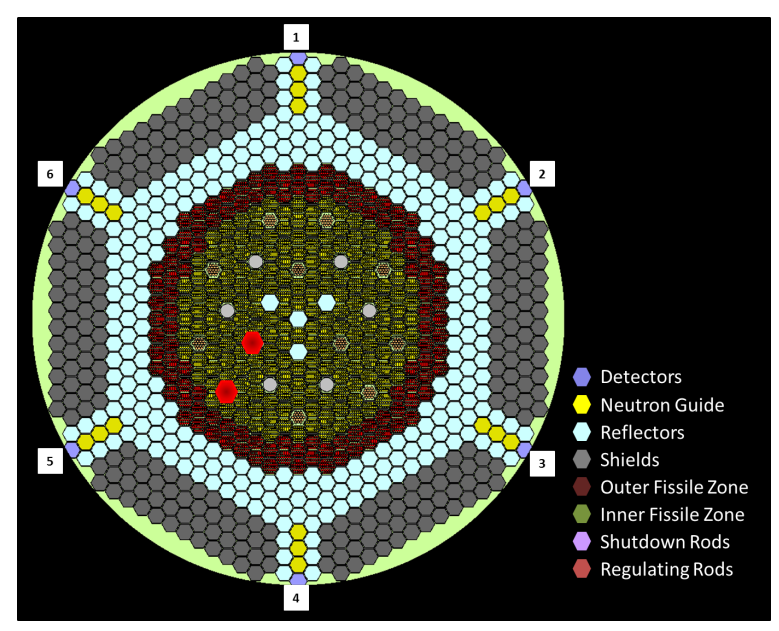

Fig. 1. Top view of SFR core.

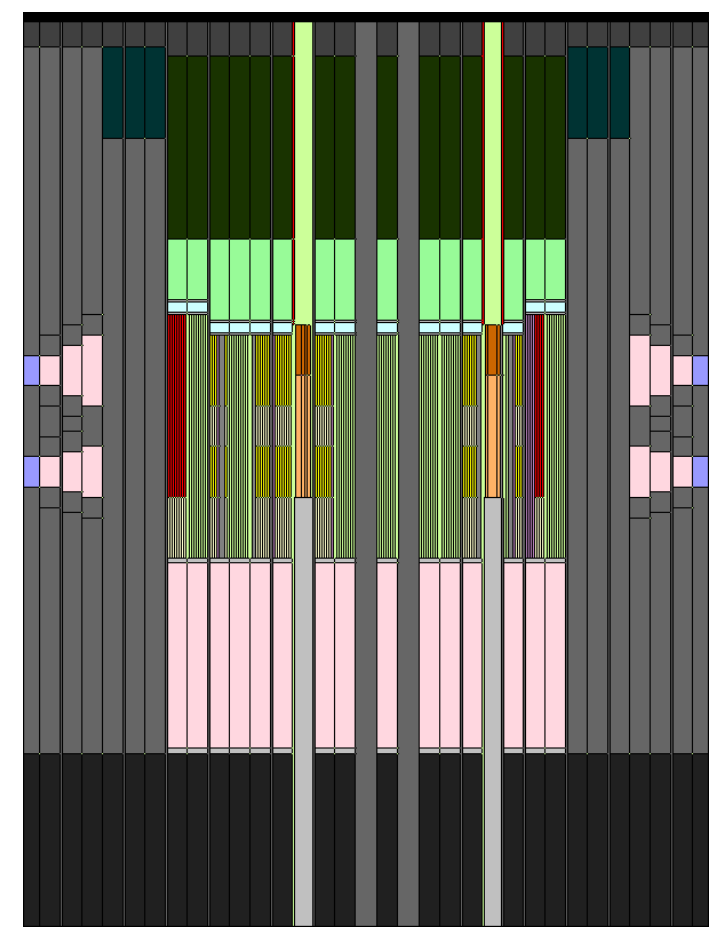

Fig. 2. Axial cross-section of the core.

upper fissile zone. Although an inadvertent control rod withdrawal (IRW) is a transient accident scenario that gradually leads to a spatially distorted neutron flux (and power level) in the core as the control rod is withdrawn, it is here modelled as a series of static problems, one for each control rod position. However, in the actual scenario, the rod removal occurs at a speed of $4 \mathrm{~mm} / \mathrm{s}$. This results in a transient that is slow enough to be considered quasi-static, and the modelling is accurate enough for the problem considered here. The reaction rates seen by the detector at location $i$ is given by

$$
R_{i}=n_{U 235} \int \sigma_{f, U 235}(E) \cdot \phi_{i}(E) \cdot d E
$$




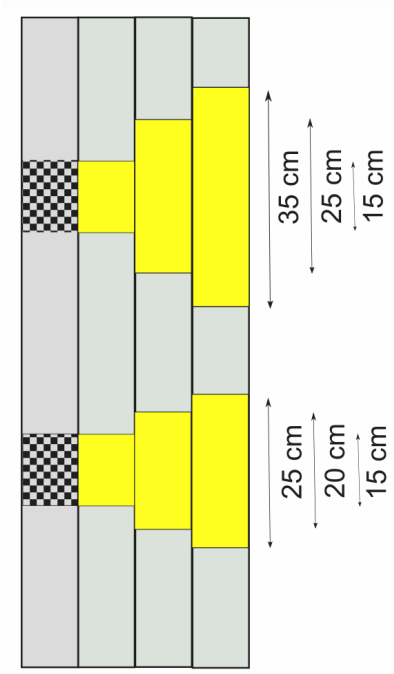

Fig. 3. Illustration of the neutron channel.

where $n_{U 235}$ is the nuclear density of the isotope U235 that constitutes the active material in the fission chamber, $\phi_{i}(E)$ is the neutron flux at the detector location, and $\sigma_{f, U 235}(E)$ is the fission cross-section of the detector material. A normalized detector response $\left(\tilde{R}_{i}\right)$ for the detector at location $i$ is then defined according to

$$
\tilde{R}_{i}=\frac{R_{i}}{\langle R\rangle}-\frac{R_{i, 0}}{\left\langle R_{0}\right\rangle}
$$

where $\langle R\rangle$ is the mean value of the 6 detectors in each ring (same plane) and the extra subscript 0 refers to the reaction rates with the control rod at the reference position. This provides a detector signal that is independent of reactor power level (and also flux normalization in the simulation), but instead monitors the angular profile changes in the core's neutron flux. Specifically, $\tilde{R}$ measures the azimuthal distortion from the chosen reference power profile of the core, $R_{0}$. Finally, a monitoring indicator $(M I)$ that tracks the control rod withdrawal is defined as the value of $A_{M I}$ that minimizes $\chi^{2}$ in the least chi-squared fit

$$
\chi^{2}\left(A_{M I}\right)=\sum_{i} \frac{\left(\tilde{R}_{i}-A_{M I} \cdot \tilde{R}_{i, 1}\right)^{2}}{\sigma_{i}^{2}}
$$

where $\sigma_{i}$ is the uncertainty of $R_{i}$ and $\tilde{R}_{i, 1}$ is the normalized response at full control rod withdrawal. An assumption is here made that $\tilde{R}$ only changes in amplitude as the control rod is withdrawn, and the shape stays constant. The confidence interval of $M I$ is then obtained from the interval of $A_{M I}$ that encloses the region $\chi^{2}<\chi_{\min }^{2}+1$. The fitting procedure to obtain $M I$ is further illustrated in the results in section in Figures 5 and 6. 


\subsection{Criticality Calculations with SERPENT}

Among several MC codes frequently used for reactor calculations, SERPENT2 was chosen for this study. SERPENT2 is a 3D continuous-energy reactor physics burn-up calculation code based on the Monte Carlo methods developed at VTT Technical Research Center of Finland. It is a newly improved version of the previous code with more emphasis on memory management and parallelization. Typically, it runs faster than several other Monte Carlo codes like MCNP5 [8,9]. Geometry building is done with a universe-based logic which nests independent structures inside one another, and neutron transport is based on a combination of conventional surface-to-surface ray-tracing and the Woodcock delta-tracking method [10]. The tracking routine has proven efficient and well suited for geometries where the neutron mean-free-path is long compared to the dimensions, $20 \mathrm{~cm}$ in this case. Serpent reads continuousenergy cross sections from ACE. We used a JEFF-3.1 based continuous energy cross-section library.

The problem considered in this paper is a source-detector response problem which is partly a shielding problem as discussed in section 2 and partly, a 3 -D criticality problem due to the asymmetrically changing neutron source profile with IRW. As the detector is far away from the core, an obvious response would be to use variance reduction techniques to provide appropriate bias to particles to get them to regions they aren't willing to go. However, our preferred approach is to treat the problem with a Monte Carlo based code in criticality mode as this approach will let us use multiple detectors at various regions in the core in a single calculation. With the variance reduction technique, one would need to perform individual calculations for each detector locations to determine appropriate bias, which can get tedious to start with. In our approach, we will be able to make better use of the neutron histories in the simulation since we include more detectors and get better statistics.

The present study focuses on beginning-of-life (BOL) condition of the core, namely a fresh core, as a starting point for Monte Carlo calculations using criticality source mode. Simulations are run in cycles and the source distribution of each cycle is formed by the fission reaction distribution of the previous one. We used constant power for our simulations. Since the core reaches an equilibrium state after long-term operation, beginning-of-cycle (BOC) state with reloading scheme is an important state to investigate after BOL. We limit ourselves to BOL calculations in this paper. 


\subsubsection{Convergence of K-eff and fission source}

Best practices for Monte Carlo calculations point out that it is essential to address certain concerns while dealing with criticality calculations [11]. For criticality calculations, $K_{\text {eff }}$ problems are based on power iteration procedure. Simulation requires an initial guess of the fission source site distribution. It is required to discard a sufficient number of initial cycles to allow the fission source distribution to converge before we can expect to have statistically relevant stable tallies. In addition, a sufficient number of neutrons must be simulated in each cycle to keep the bias in $K_{\text {eff }}$ and reaction rate tallies small enough to be able to trust the results.

Also, it is imperative to know when the fission source has converged as it is not just sufficient to show the convergence of $K_{\text {eff }}$ alone. $K_{\text {eff }}$ may converge before the fission distribution for Monte Carlo criticality problems. Given that the Shannon entropy of the fission distribution is scalar, it is a convenient means of characterizing the convergence of a fission source [11]. Evaluation with Shannon entropy is based on the idea of superimposing a $3 \mathrm{D}$ grid on all fissionable regions and tallying the number of fission sites in a batch that fall into each of these cells. These tallies give an estimate of the fission source spatial distribution.

$$
H_{\text {source }}=E\left[I\left(P_{k}\right)\right]=E\left[-\log _{2}\left(P_{k}\right)\right]=-\sum_{k=1}^{N_{b}} P_{k} \cdot \log _{2}\left(P_{k}\right)
$$

where $N_{b}$ is the number of grid cells in the superimposed mesh and $P_{k}$ is the number of source sites in $k$ - th grid box divided by the total number of source sites. One recalls that Shannon entropy is the expected value of the information $I\left(P_{k}\right)$, which is equal to $-\log _{2}\left(P_{k}\right)$ since it is a positive and additive quantity [12]. Monte Carlo codes compute the entropy of the fission source distribution which is given as a batch by batch evolution and is used to guess the fission source stationarity.

For our case, the trial population size per cycle is $5 \cdot 10^{6}$ and the total number of active cycles are 10000 which makes the total neutron histories equal to $5 \cdot 10^{10}$. We checked the convergence of both $K_{\text {eff }}$ and fission source distribution.

\section{Results}

The distortion of the neutron flux profile, and hence the power profile, during an inadvertent control rod withdrawal is illustrated in Figure 4, which shows contour plots of a 280 by $280 \mathrm{~cm}$ mesh-tally in the SERPENT simulation. The mesh is placed at the core mid-plane, and the units are $10^{15} \mathrm{~cm}^{-2} \mathrm{~s}^{-1}$. In 

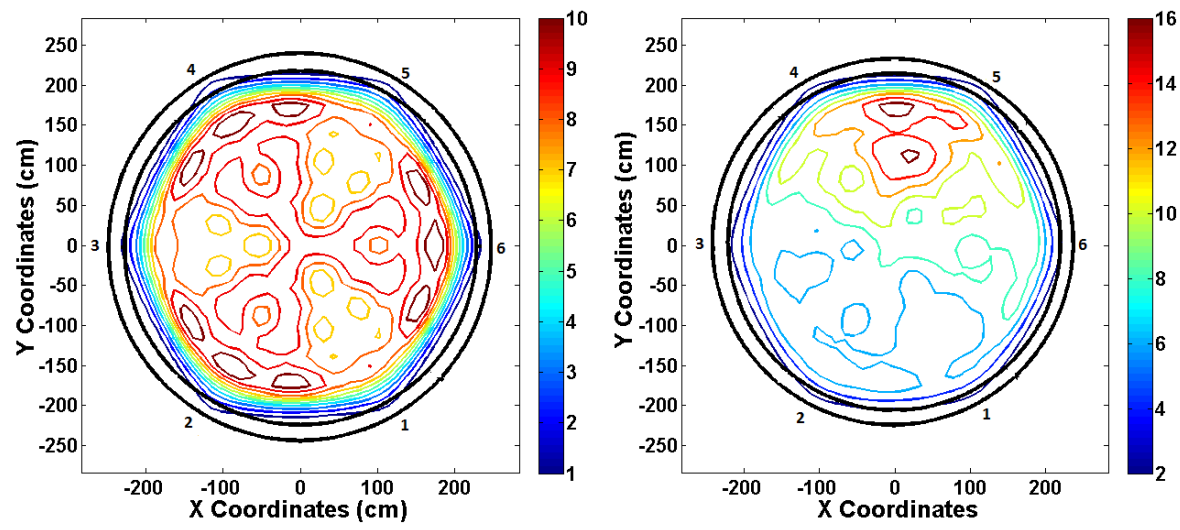

Fig. 4. Response function of U-235 at core mid-plane in reference and withdrawn case respectively.

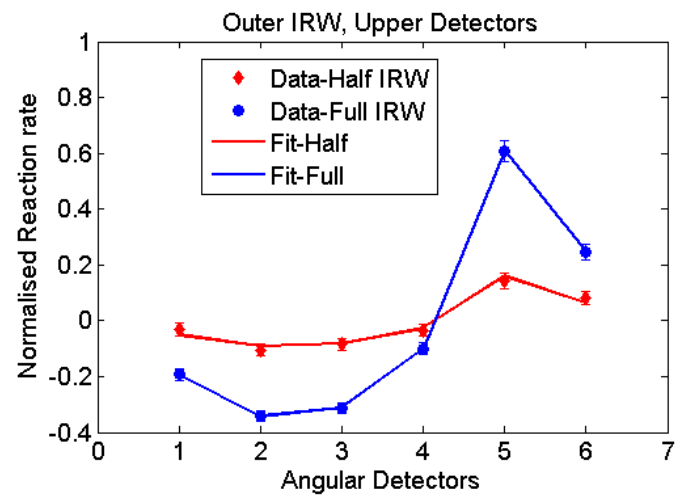

(a) Upper Detector

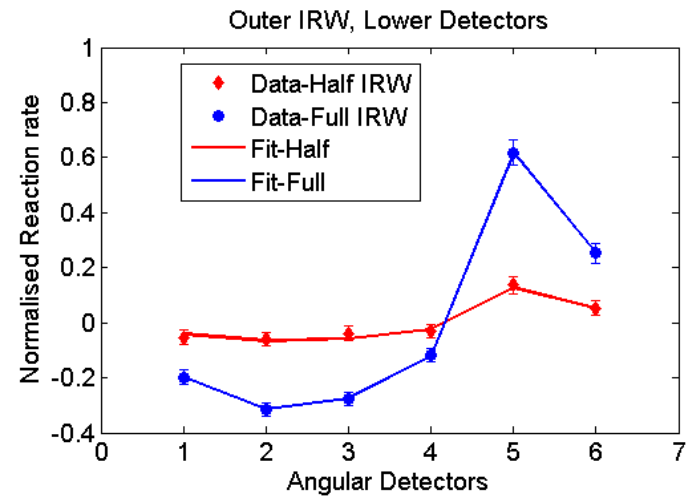

(b) Lower Detector

Fig. 5. Relative Response in case of Full IRW for Outer IRW

the left panel the neutron flux is shown for the control rod in the reference position, and in the right panel with one control rod fully withdrawn. The latter shows a strong angular asymmetry as expected. The locations of the 6 angular detectors from figure 1 are given as well.

In Figure 5(a), the normalized reaction rates for the detectors are presented with the results from the upper detectors and in 5(b), the results from the lower detectors. Full control rod withdrawal is shown in blue and half withdrawal is shown in red. The points with error bars show the normalized reaction rates $\left(\tilde{R}_{i}\right)$ for each individual fission chamber, and the solid lines show the results of the best fits of $A_{M I}$ according to equation 3. Figure 6, using a different vertical scale, shows the corresponding information for the withdrawal of an inner control rod.

Finally, in Figure 7 the monitoring indicator $(M I)$ derived from the fits is shown as a function of control rod withdrawal. The upper detectors are shown 


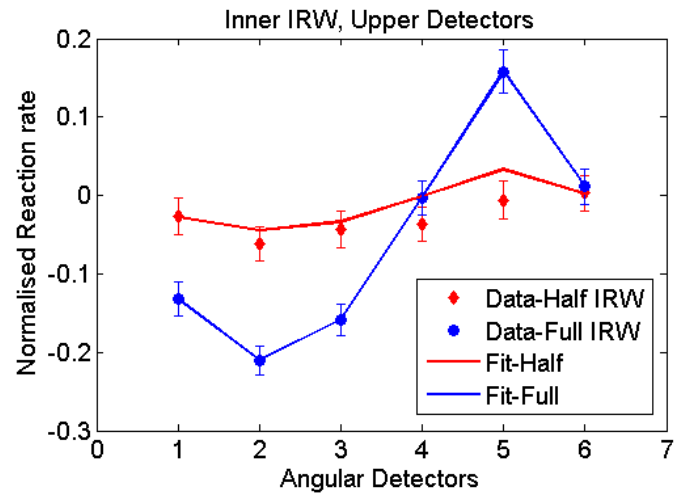

(a) Upper Detector

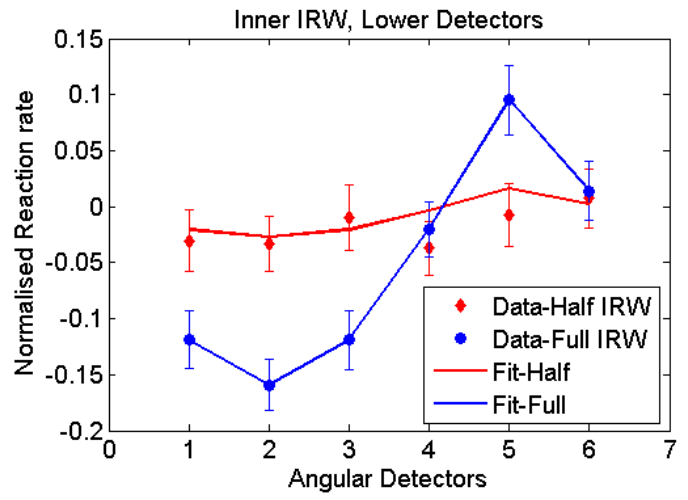

(b) Lower Detector

Fig. 6. Relative Response in case of Full IRW for Inner IRW

in red, and the lower detectors are shown in blue. It should be noted that the results for $0 \%$ and $100 \%$ withdrawal are 0 and 1 according to the definition of $M I$ in equation 3. They are however included for completeness. The solid lines are shown as guides for the eye.

Starting with an outer control rod withdrawal (7 (a)), there is a small but statistically significant difference between $M I$ in the upper and lower detector sets. For both detector sets there is a close to quadratic relationship between $M I$ and the control rod position. The upper detector set gives an earlier response in $M I$ during a control rod withdrawal than the lower detector set. The statistical uncertainty in $M I$ for both sets is about \pm 0.03 . The results for $M I$ from an inner control rod withdrawal are similar, but less pronounced. The statistical uncertainty in $M I$ is here \pm 0.11

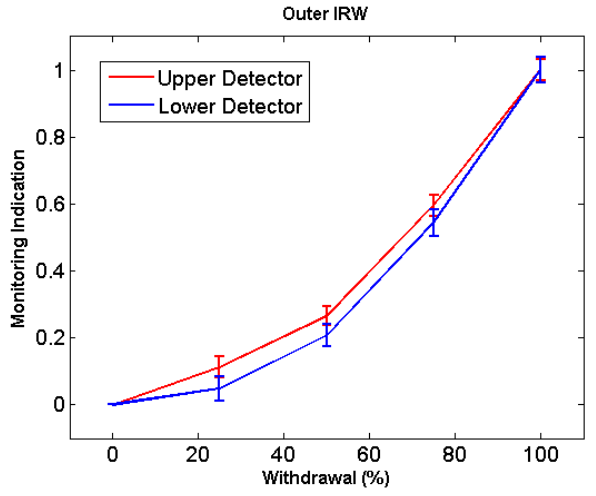

(a) Outer IRW

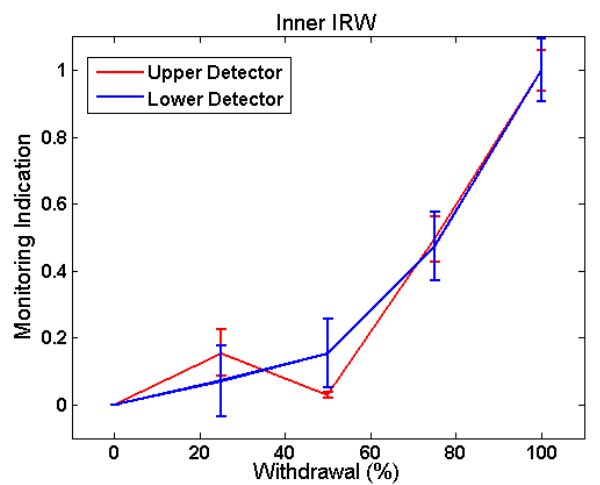

(b) Inner IRW

Fig. 7. Monitoring Indication for Outer and Inner IRW 


\section{Discussion}

In this paper, we used two sets of 6 equally-spaced fission chambers, each located in a different axial plane, to monitor azimuthal distortion in the power profile of a sodium cooled fast reactor. The detectors are installed beyond the lateral neutron shield, which places them in-vessel providing a strong enough signal, but outside the core. Due to the very compact design of an SFR, it is not deemed feasible to install fission chambers inside the core. We introduced a tapered neutron channel in the lateral shield to provide a collimated path to the detectors and further increase their signal.

A scenario that would result in a non-symmetric distortion of the neutron flux is a withdrawal of a single control rod. This in turn leads to a nonsymmetric signature in the ring of fission chambers located around the core. In the paper we employ a monitoring indicator $(M I)$ that quantifies the difference between the detector signals when the core is in its reference state and the corresponding signals when the core has one of its control rods partially or fully withdrawn. In the case of an outer control rod withdrawal, the statistical uncertainty of $M I$ derived from the simulations presented in this paper is about $\pm 3 \%$. From the results presented in Figure 7(a), a withdrawal of about $5 \%$ for an outer control rod would be enough to yield a significant signature in the $M I$. To arrive at an $M I$ with $3 \%$ uncertainty in the simulation required a statistical uncertainty in the reaction rate of each detector $\left(R_{i}\right)$ of about $\pm 2 \%$. For a real fission chamber, this is by no means an unrealistic assumption. The results presented here should therefore be representative of what could be done in real installation at a reactor.

However, for long term operations one could imagine drifts in the fission chambers' relative calibrations that are larger than $2 \%$, which in turn would result in a false change in the measured power profile in the detector ring. This is an issue that is addressed with using a reference profile $\left(R_{0}\right)$ in equation 2 . If one looks for sudden changes in the power profile, e.g. on the order of the withdrawal time of a control rod, $R_{0}$ would be continuously updated with the measured profile from a few minutes ago. On the other hand, if one looks for slower changes in the power profile, a reference $R_{0}$ from a week ago could be used. In both cases the fission chambers in the angular ring would be automatically re-calibrated relative to each other.

For the studied reactor core, we show that it is possible to detect withdrawal of an outer control rod at an early stage. The particular control rod being withdrawn can be identified from the measured distorted flux profiles. However, for an inner control rod withdrawal the angular detectors are less efficient. For this reason, one additional, centrally located in-core detector would be needed. As mentioned above, fission chambers are not suitable for this location, but 
one detector type that could potentially be used inside an SFR core is the self-powered neutron detector (SPND). Such detectors are typically used in Light Water Reactors for in-core monitoring. Investigating the potential for using an SPND in fast reactor applications will be the focus of a forthcoming work.

The fact that the calculations were done in criticality mode results in a comparatively long calculation time. The large size of the core and the fact that the fission chambers are situated behind the neutron shield results in a low fraction of neutron histories reaching the detectors. The calculations must therefore be run with a relatively large number of total neutron histories compared to calculations in source mode using variance reduction. However, given the computing power available today, this is not a major limitation. During the initial design phase of a neutron monitoring system, one needs to explore various locations and configurations. A single criticality calculation lets us analyze detection rates at multiple locations without having to redo the simulations for each configuration. The criticality calculations can give the general trends for monitoring indications which can be useful in qualifying a certain region as a possible detector location without using variance reduction techniques at the very beginning. For example, in this study we could identify the upper region as a more suitable location for the angular detectors.

\section{Conclusion}

We investigated the possibility of using a set of in-vessel, ex-core fission chambers to detect changes in the neutron source distribution initiated by inadvertent control rod withdrawal. The results showed that it is possible to detect the withdrawal of control rod in the core with six equally-spaced detectors in a single plane. To be able to precisely know the location of perturbation in the core, we need to have robust core monitoring in the radial direction. We suggest the use of in-core detectors coupled with the peripheral fission chambers.

\section{Acknowledgements}

The study was performed in the framework of an on-going collaboration on the instrumentation and safety of sodium cooled fast reactors between Uppsala University and CEA with support from the Swedish Research Council. 


\section{References}

[1] C. Jammes, N. Chapoutier, P. Filliatre, J. P. Jeannot, F. Jadot, D. Verrier, A.C. Scholer, B. Bernardin, Neutron flux monitoring system of the french GEN-IV SFR: Assessment of diverse solutions for in-vessel detector installation, Nuclear Engineering and Design 270 (0) (2014) 273-282.

[2] P. Filliatre, C. Jammes, B. Geslot, L. Buiron, In vessel neutron instrumentation for sodium-cooled fast reactors: Type, lifetime and location, Annals of Nuclear Energy 37 (11) (2010) 1435-1442. doi:10.1016/j.anucene.2010.06.023.

[3] F. Varaine, P. Marsault, M.-S. Chenaud, B. Bernardin, A. Conti, P. Sciora, C. Venard, B. Fontaine, N. Devictor, L. Martin, A.-C. Scholer, D. Verrier, Preconceptual design study of ASTRID core, in: Proceedings of ICAPP 12 - Int. Congress on Advances in Nuclear Power Plants, no. 12173, Chicago, USA, 2012.

[4] J. Leppänen, SERPENT users manual, Tech. rep., VTT (2012).

URL http://www.montecarlo.vtt.fi

[5] C. Jammes, P. Filliatre, B. Geslot, L. Oriol, F. Berhouet, J. Villard, L. Vermeeren, Research activities in fission chamber modeling in support of the nuclear energy industry, IEEE Transactions on Nuclear Science 57 (6) (2010) 3678 -3682. doi:10.1109/TNS.2010.2075939.

[6] C. Jammes, P. Filliatre, B. Geslot, T. Domenech, S. Normand, Assessment of the high temperature fission chamber technology for the french fast reactor program, IEEE Transactions on Nuclear Science 59 (4) (2012) 1351-1359.

[7] Z. Elter, C. Jammes, I. Páazsit, L. Páal, P. Filliatre, Performance investigation of the pulse and campbelling modes of a fission chamber using a poisson pulse train simulation code, Nuclear Instruments and Methods in Physics Research Section A: Accelerators, Spectrometers, Detectors and Associated Equipment 774 (2015) 60 - 67. doi:http://dx.doi.org/10.1016/j.nima.2014.11.065.

[8] J. Leppänen, Standard comparison between SERPENT 1.1.13 and MCNP5, Tech. rep., VTT (2010).

URL http://montecarlo.vtt.fi

[9] X-5 Monte Carlo Team, MCNP - A General Monte Carlo N-Particle Transport Code, Version 5 (Los Alamos National Laboratory, Los Alamos, New Mexico, USA, April), Tech. Rep. LA-UR-03-1987, Los Alamos National Laboratory (LANL) (2003).

[10] E. R. Woodcock, et al., Techniques Used in the GEM Code for Monte Carlo Neutronics Calculations in Reactors and Other Systems of Complex Geometry, in: Proceedings of the Conference on Applications of Computing Methods to Reactor Problems, Argonne National Laboratory, 1965.

[11] F. B. Brown, On the use of shannon entropy of the fission distribution for assessing convergence of monte carlo criticality calculations, in: PHYSOR-2006 
American Nuclear Societys Topical Meeting on Reactor Physics, Vancouver, BC, Canada, Sept. 1014, 2006.

[12] C. Shannon, A mathematical theory of communication, The Bell System Technical Journal 27 (3) (1948) 379-423. doi:10.1002/j.1538-7305.1948.tb01338.x. 